Université de Ljubljana

\title{
LA DETERMINATION NOMINALE: ARTICLE ET QUANTIFICATEUR
}

Le présent article se propose d'examiner certaines occurrences de syntagmes nominaux et plus particulièrement de détermination nominale. L'accent sera mis sur quelques cas susceptibles de répondre en partie aux exigences d'un apprenant slovène puisque la détermination en slovène se manifeste entre autres à l'aide des déclinaisons ou des prépositions et non de l'article. Il s'agira essentiellement de mettre en évidence l'opposition article et quantificateur.

\section{Introduction}

Les déterminants du nom sont définis de manière classique comme les unités fonctionnelles qui, antéposées au nom, permettent de construire un syntagme nominal minimal constituant la phrase. Ainsi "un" ou "les" sont des déterminants puisqu' on peut avoir "un chien aboie" ou "les chiens" tandis que la construction "méchant chien aboie" est jugée agrammaticale en français ("méchant" ne faisant pas partie de cette classe). L'énoncé "un chien aboie" est certes ambigu car il peut désigner l'animal en train d'aboyer ou un type de cri appartenant à la classe des chiens. Le contexte permet en général de lever l'ambiguïté. Parfois, le syntagme nominal peut être construit sans déterminant, mais il s'agit des cas de non-détermination qui ne feront pas l'objet de cette courte étude.

Afin de parer aux nombreuses fautes relatives à l'emploi des déterminants et de sensibiliser les apprenants slovènes à l'usage des différents déterminants, nous avons choisi d'aborder quelques formes sujettes à confusion ou parfois peu analysées. Partant d'une étude de cas établie par A. Winther, qui analyse les occurrences du roman de J. Ricardou Les Lieux-dits, et sans pour autant être exhaustif, nous avons mis en valeur voire développé les problèmes qui nous intéressent plus particulièrement et relèvent d'une première distinction à établir en matière de détermination: l'opposition "article" et "quantificateur".

\section{Les titres}

Si certains titres ne comportent pas et ne sauraient comporter de déterminant comme "Histoire de France", d'autres en revanche, comme "Le Rouge et Le Noir" ou "Un amour de Swann", ne peuvent s'en passer. Ainsi, les titres qui semblent s'opposer, et sont sans déterminant, sont les titres déterminés d'oeuvres littéraires (à moins qu'il ne s'agisse de noms propres comme "Lucien Leuwen" ou "Eugénie Grandet"), les titres de manuels et les oeuvres dites cognitives comme les dictionnaires et les encyclopédies. Or, ce n'est pas toujours le cas: par exemple, si “Dictionnaire du Français 
contemporain" ne prend pas de déterminant, le titre "Les nominalisations en français" comprend un syntagme déterminé.

On distingue donc deux fonctions du syntagme nominal. L'ouvrage intitulé "Dictionnaire du Français contemporain " est un dictionnaire en ce sens qu'il est bien une représentation du lexique de la langue française contemporaine au même titre que le livre intitulé "Histoire de France" contient l'histoire de la ou des périodes concernées. Ainsi, lorsque le titre donne un cadre général, on assiste à l'omission de l'article. Pris dans ce sens, le titre peut être comparable à une enseigne comme "Boulangerie-Pâtisserie". Aussi, le syntagme non déterminé en nombre est le prédicat qui identifie la nature du référent qu'il accompagne si bien que l'absence de pré-déterminant est souvent la marque de la fonction prédicative du syntagme nominal.

Par ailleurs, si on substitue à "Nominalisations en français", le syntagme "Les nominalisations en français", on s'attend à trouver sous ce titre non pas un livre de grammaire mais bien une étude sur les nominalisations en français. De même "Le Rouge et le Noir" n'est pas un livre sur les couleurs "rouge" et "noire" mais une oeuvre qui raconte une histoire qui se passe sous la Restauration et le livre "Les Lieux-dits" n'est pas un "livre de lieux-dits", car cela n' existe pas, mais un ouvrage qui traite de certains lieux-dits. Par conséquent, le titre déterminé n'est plus le nom du référent qui le porte mais il est en relation syntagmatique avec le texte qu'il caractérise. C'est en quelque sorte la première phrase du texte. La détermination crée donc bien la référence qui est, dans le cas présent, une référence contextuelle.

\section{Le nom propre dans les titres}

Le nom propre (de lieu, de personne, etc.) désigne un signe linguistique particulier, propre à un seul référent. Il se distingue du nom commun dont le signifiant donne lieu à un concept ou à un signifié qui permet souvent de désigner une classe de référents. Le nom propre n'a pas de signifié mais il prend la fonction de signifiant d'un référent. La détermination sert donc à créer la référence: si le mot "chien" ne désigne pas un référent, au contraire, " $u n$ chien, $c e$ chien, mon chien" déterminent une référence précise. Ainsi, d'une manière générale, les noms propres n'ont pas besoin de référence puisqu'ils sont le signe d'un référent unique.

Cependant, de nombreux noms propres comprennent un déterminant qui est souvent l'article défini. Il s'agit des noms propres patronymes comprenant un article contracté comme "Leroux" ou "Dupont" ou encore des noms propres toponymes comme "Le Havre" ou "Le Val de Marne". Ces noms propres sont issus de formations régulières qui relèvent souvent d'une description ou d'une désignation définie. On distinguait ainsi autrefois, dans un village, les personnes qui portaient le même nom de baptême: Paul le roux par opposition à un autre Paul le brun. Ou encore, on distinguait Pierre qui habitait à côté dudit pont ou dudit val etc. Ces formations ont donné par la suite des noms figés en patronymes comme Lebrun, Leroux ou Dupont, Duval etc. Le même fonctionnement s'observe pour le nom de certains rois comme Saint Louis, 
Charles le Chauve ou Charles le Bel. Ainsi, l'article permet d'identifier un référent unique en renvoyant à une caractéristique de ce référent. On parle également d'article notoire pour désigner ce fonctionnement de l'article défini.

Par ailleurs, il s'agit de ne pas confondre cette distinction de référent par la description d'une caractéristique notoire avec l'usage de l'article dans le syntagme suivant, "le rusé Ulysse" ou "le Balzac collectionneur" qui marque une syntaxe différente. L'emploi d'un adjectif épithète antéposé ou postposé avec un nom de personne nécessite en général l'emploi de l'article. Il ne s'agit plus de distinguer un Ulysse ou un Balzac parmi tant d'autres mais dans ce cas, l'épithète note un trait caractéristique du personnage ou du héros. La syntaxe est donc celle d'un nom commun et si l'article y est encore notoire, la place de l'adjectif est caractéristique d'une référence admise et non pas d'une caractéristique latente, connue ou manifeste.

Les toponymes déterminés peuvent également être issus de noms communs dans lesquels l'article défini possède la même fonction que dans "le soleil" ou "la terre". Le référent est unique et l'article défini est là encore notoire. Il renvoie à un référent bien connu. Si le référent n'est pas connu de tous, une détermination supplémentaire s'impose comme "Le Gros Theil" par exemple.

Par conséquent, le nom propre est le signifiant d'un référent unique qui peut être arbitraire et admis par la convention ou encore il peut s'agir d'une description définie, qui a été construite à l'aide d'un nom commun et de l'article défini notoire mais désigne également un référent unique.

\section{Le déterminant indéfini}

Si l'article défini indique souvent un référent précis, connu ou spécifique, l'article indéfini "un" ou "une" est le déterminant indéfini ("un quelconque") applicable aux référents comptables. Pour les référents non-comptables, une forme de suppléance de déterminant indéfini " $\mathrm{du}$, de la" a été créée. Il s'agit d'une forme particulière de quantifieur peu précis ou flou. Ainsi, on peut, d'une manière générale, représenter le système des formes et des valeurs des déterminants de la façon suivante:

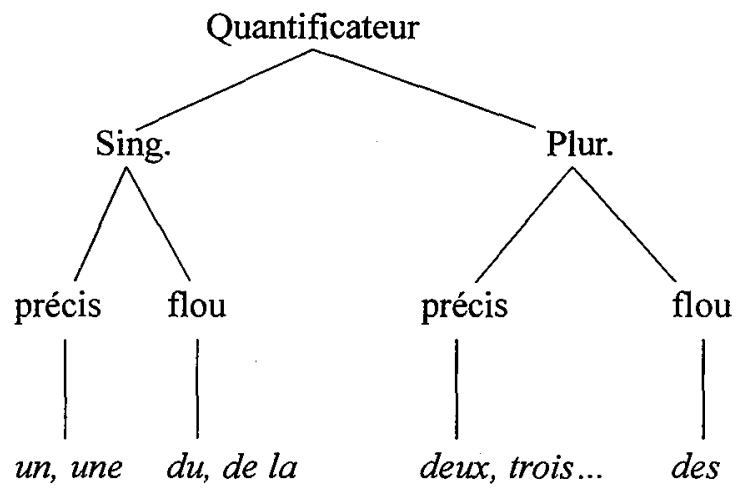


Cependant, quelques remarques s'imposent. En effet, tous les noms ne font pas partie de l'opposition comptable/non-comptable. Ainsi, le mot "rosbeef" peut désigner la substance ou la catégorie de viande dans la phrase: "manger du rosbeef". Mais, il peut également désigner la portion de viande taillée dans un morceau: "manger un rosbeef". Il en va de même pour les mots comme "la tarte, le rôti ou le bifsteack etc." Par ailleurs, un nom peut passer d'une catégorie à l'autre, c'est-à-dire du comptable au non-comptable: "élever des poules" concerne la masse de poules tandis que dans la locution, "produire du poulet en batterie", le poulet est considéré non pas comme un volatile mais comme un produit industriel contrairement à "un coq chante" qui désigne le volative ou son cri voire les symboles qui apparaissent à l'image du coq. Inversement, on peut passer de la catégorie non-comptable au comptable: "ce pays produit du vin" et "ce pays produit un vin de qualité" où la détermination fait passer le produit ou la substance à une espèce nombrable. On peut également ranger dans cette catégorie les nombreux emplois implicites largement usités au quotidien: "j'ai acheté une eau" pris pour "une bouteille d'eau" voire les quantifications métonymiques comme "faire les cuivres" qui mêlent la substance et l'objet.

Par conséquent, on peut considérer que l'article indéfini complète l'article défini lorsqu'il permet un mode de référence différent. Par exemple, si deux personnes sont à la recherche d'un objet et l'une d'elles s'exclame: "la lettre !", on comprendra qu'elle est à la recherche de cette lettre dont on connaît l'existence. Au contraire, si elle s'écrit: "une lettre !", on peut en conclure que la présence d'une lettre est un élément nouveau à cet endroit. Aussi, l'article défini signale du connu et un référent dont l'existence n'est pas ou ne peut pas être mise en cause et ainsi être une source d'information pour l'allocutaire. On peut le considérer comme un support d'information. Au contraire, l'article indéfini désigne des occurrences non-spécifiées du référent signifié c'est-àdire non-connues, nouvelles ou autres que les occurrences déjà mentionnées. Il signale un référent dont l'existence est une information en soi. C'est donc un apport d'information.

Cependant, si l'article défini désigne en situation ou renvoie en contexte, faisant ainsi partie des déterminants dits déictiques, ce n'est pas le cas du déterminant indéfini qui ne peut désigner qu'un seul référent: "il était une fois, une princesse qui... Un jour, un prince vint...". Aussi, si l'article indéfini est un non-déictique, on peut penser qu'il s'oppose en ce sens au paradigme des articles (défini, démonstratif, possessif etc.) dont il ne possède pas les caractéristiques pour devenir un quantificateur (au même titre que "plusieurs, quelques, peu de" etc.) c'est-à-dire qu'il prend les caractéristiques du déterminant numéral.

Le paradigme des articles et celui des quantificateurs sont en relation syntagmatique puisqu'on les utilise ensemble comme l'indiquent les exemples suivants: "ces quelques fleurs" ou "les quatre cartes". Mais l'indéfini "des" ne sera jamais en position d'article dans ces mêmes phrases: on ne peut avoir "*des quelques fleurs" ou "*des quatre cartes". Il y a donc complémentarité entre les deux déterminants au plu- 
riel: l'article marque le thème et la pluralité tandis que le numéral détermine la pluralité en la quantifiant, d'où le nom de "quantificateur". On peut également noter cela de la manière suivante:

Dét. ART. + QUANT

On distingue donc au moins deux paradigmes de déterminants: le paradigme des articles, caractérisé par une fonction déictique et un fonctionnement thématique (support) d'une part et, d'autre part, le paradigme des quantificateurs qui comprend l'article indéfini pris au sens d'indéfini numéral cardinal et quantifie, de manière précise, les noms comptables (un, deux, trois... livres) mais aussi les formes partitives qui, de leur côté, quantifient de manière floue les noms non-comptables ( $d u$ cuivre, de la bière). La quantification peut donc être considérée comme la forme première ou minimale de la détermination nominale.

Les articles se construisent aussi bien avec des noms comptables (la table) que des noms non-comptables (le lait) tandis que les déterminants quantificateurs sélectionnent les noms comptables (une table, deux chaises), à l'exception des quantificateurs partitifs flous "du, de la" qui déterminent les noms à référent non-comptable (du lait, de la soupe). Les quantificateurs ne peuvent déterminer les noms comptables que si une modification sémantique du syntagme suit (une eau, pris pour une quantité d'eau).

Comparons donc les caractéristiques de l'article "le" par rapport aux quantificateurs "un" et "du":

\begin{tabular}{|c|c|c|}
\hline \begin{tabular}{|l} 
E: \\
articlerminant \\
défini \\
singulier \\
quantité précise \\
référent comptable \\
ou non-comptable \\
thème ou support
\end{tabular} & $U N: \mid \begin{array}{l}\text { déterminant } \\
\text { quantificateur } \\
\text { numéral / indéfini } \\
\text { singulier } \\
\text { quantité précise/ floue } \\
\text { référent comptable } \\
\text { rhème ou apport }\end{array}$ & $D U: \mid \begin{array}{l}\text { déterminant } \\
\text { quantificateur } \\
\text { partitif } \\
\text { singulier } \\
\text { quantité floue } \\
\text { réf. non-comptab } \\
\text { rhème ou apport }\end{array}$ \\
\hline
\end{tabular}

\section{Les déterminants partitifs}

Prenons les exemples suivants: "Il a besoin $d u$ stylo, de la gomme et des ciseaux" par opposition à la phrase suivante, "Il mange de la salade, $d u$ rôti et des frites". On remarque que la locution "avoir besoin" est un verbe transitif indirect construit à l' aide de la préposition "de". Cette préposition précède l'article au féminin, est contractée avec l'article défini au pluriel et au masculin singulier tandis que "manger" est un verbe transitif direct, dont la construction même ne requiert pas de préposition. Il s'agit donc, dans ce deuxième exemple, de l'emploi des formes évoquées précédemment ("du, de la" et "des", pluriel de "un") qui incluent "de", comme l'indéfini "des". Ces formes sont généralement qualifiées d'article partitif. 
Ces formes sont morphologiquement homonymes des formes de l'article défini contracté précédé de la préposition "de", même si elles sont différentes. Par ailleurs, signalons que l'indéfini "des" a un singulier "un, une", tandis que les déterminants partitifs "du, de la" ont un pluriel morphologique mais n'ont pas de pluriel sémantique. En effet, "manger de la tarte" indique qu'on a mangé une partie indéterminée de la tarte tandis que "manger des tartes", c'est manger un certain nombre indéterminé du met "tarte".

On note donc l'opposition entre référents comptables et non-comptables: le mot "stylo" est une unité lexicale renvoyant à un référent-objet comptable et s'emploie donc de manière précise et sans problème particulier au pluriel (cinq stylos) ou, de manière floue (des stylos). Au contraire, "salade ou bière" désignent non pas des individus ou des objets, comme dans l'exemple précédent, mais des substances ou des matières, non précises, continues et indistinctes et renvoient à des référents non-comptables ou concepts.

Aussi, ces référents non-comptables ne peuvent s'employer en tant que tels au pluriel: en effet, "une assiette de *salades" ou "un château de *sables" sont incorrects. Ces mots ne peuvent être utilisés avec les quantificateurs "des" qui impliquent un référent précis et comptable et non pas un référent continu et non-comptable si bien que le rôle de la préposition "de" est de fragmenter le référent en faisant référence à une partie de la substance ou de la matière et non pas à sa totalité en tant que référent continu. Sa fonction sera non pas de quantifier mais de quotifier c'est-à-dire d'exprimer la quotité de la quantité ou une indication quantitative, même de manière floue et imprécise.

On peut cependant quantifier de manière précise en ayant recours à des indicateurs de quantité (poids ou volumes): "une bouteille de bière" ou "une noix de beurre". On peut également rester dans une quantité imprécise et floue et employer une forme combinant le "de" partitif au singulier de l'article défini notoire: "de la bière" correspond à une partie indéterminée de la substance ou boisson "bière".

Nous avons déjà noté que certains noms peuvent également faire partie des deux catégories comptables et non-comptables comme "manger de la tarte "et "manger une tarte". De même, certains mots peuvent passer d'une catégorie à l'autre ou encore avoir recours à une quantification implicite.

Récapitulons nos propos sous la forme d'un tableau:

\begin{tabular}{|c|c|c|c|}
\hline \multicolumn{3}{|c|}{ quantificateur } \\
\hline \multicolumn{2}{|c|}{ singulier } & \multicolumn{2}{c|}{ pluriel } \\
\hline précis & flou & précis & flou \\
\hline Un, une & Du, de la & Deux, trois... & des \\
\hline
\end{tabular}

\section{Les déterminants DES}

Il convient de ne pas confondre les deux formes homonymes "des" dont une des formes désigne le pluriel du déterminant indéfini singulier "un" (admirer des maisons) 
et l'autre forme désigne le pluriel de la forme contractée en "de" de l'article défini (index des auteurs).

On note, par ailleurs, une opposition dans l'usage de ces deux formes: l'exemple "des maisons" constitue un référent nouveau et indéfini qui n'a pas encore été introduit tandis que "des auteurs" s'attache à des référents connus et définis et caractérise les noms des auteurs cités dans l'ouvrage. La présentation suivante indique clairement cette opposition. Dans le premier cas, on remarque l'existence du syntagme nominal prépositionnel et de la préposition "de":

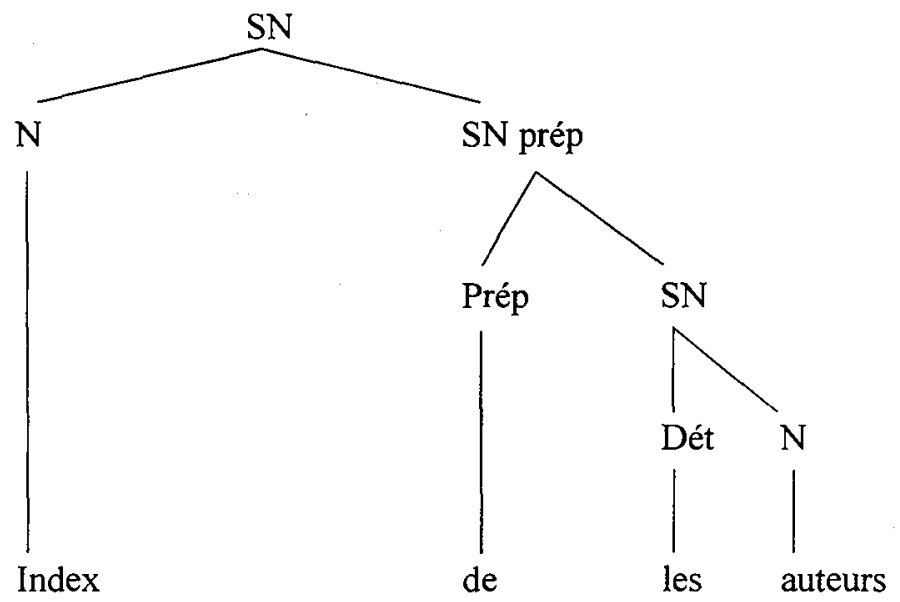

(des)

( $\mathrm{SN}=$ syntagme nominal; $\mathrm{N}=$ nom; Prép = préposition; Dét = déterminant $)$.

Dans le second cas, le verbe "admirer" ne requiert l'emploi d'aucune préposition: "des" est le déterminant puisqu'il peut être remplacé, par exemple, par "certaines, quelques, plusieurs etc.":

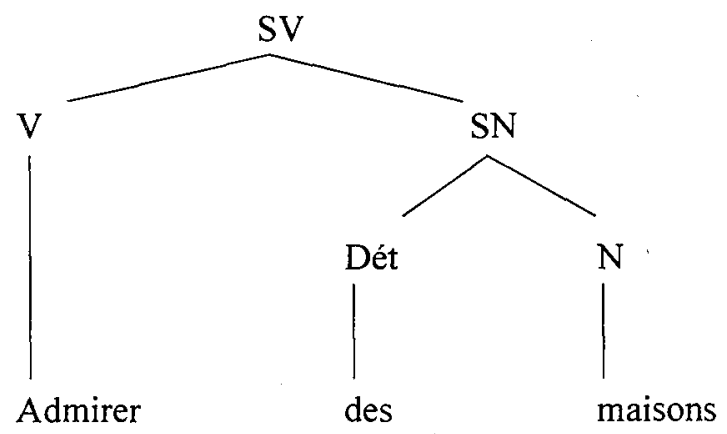

( $\mathrm{SV}=$ syntagme verbal; $\mathrm{V}=$ verbe $; \mathrm{SN}=$ syntagme nominal; $\mathrm{N}=$ nom; Dét = déter minant). 
Par conséquent, il s'agit de bien distinguer deux formes qui s'opposent au niveau du référent et de la syntaxe mais dont la morphologie est la même. D'une part, l'article indéfini "un" correspond bien au numéral cardinal du paradigme des quantificateurs énoncés auparavant "un, deux, trois...livres" et est employé en tant que déterminant principal. Il désigne un nombre de référents flous ou quelconques du concept désigné par le substantif auquel il se rapporte: "un livre" correspond au référent flou ou quelconque de 'livre'. Aussi, il ne s'agit pas de deux formes homonymes distinctes mais d'une même forme de "un" qui est à la fois un numéral (valeur numérale) et un déterminant indéfini (valeur indéfinie).

La forme "un" possède donc plusieurs pluriels, c'est-à-dire des formes signifiant des quantités définies et précises (valeur ou quantité numérale définie et précise) comme dans l'exemple "index des auteurs" puisqu'il s'agit d'un index qui précise le noms des auteurs. On retrouve, par ailleurs, l'article défini précédé d'une préposition, syntaxiquement extérieure au déterminant mais morphologiquement intégrée. Dans l'exemple suivant, "admirer des maisons", la forme "un" possède aussi des quantités indéfinies et floues (valeur et quantité indéfinie et floue) puisqu'il s'agit de n'importe quel référent de maison. Récapitulons cela sous forme de tableau:

\section{UN}

\begin{tabular}{|l|l|l|l|}
\hline \multicolumn{2}{|c|}{ article défini } & \multicolumn{2}{c|}{ déterminant numéral } \\
\hline - non-contracté & - contracté avec “de" & & \\
- singulier et précis & - singulier et précis & - singulier/pluriel & - pluriel \\
- pluriel et flou & - pluriel et flou & - précis & - flou \\
- le, la/les & $-d u$, de la/des & $-u n$, une/deux, trois... & - des \\
\hline
\end{tabular}

\section{L'opposition DE/DES}

Le dernier point que nous traiterons est déterminé par la forme "de": "il y a des roses jaunes dans le jardin" et non pas "il y a *de roses jaunes dans le jardin" par rapport à "il y a de/des belles roses dans le jardin". Le quantificateur "un" a deux formes de pluriel "des" et "de" lorsque le syntagme nominal comprend un adjectif antéposé au nom. Or, si les deux formes "des" et "de" peuvent s'employer devant l'adjectif "belles", "de" ne peut apparaître que devant un adjectif qui a été modifié comme "de très belles roses" et non "*des très belles roses", comme on peut l'entendre parfois. On peut supposer que la forme archaïque et littéraire "* des belles fleurs" demeure par opposition à la forme actuellement admise "de belles fleurs" où la forme "de" joue le rôle d'article partitif tandis que la forme archaïque suppose l'emploi du quantificateur. Rappelons également la règle classique relative à l'omission du déterminant devant un adjectif antéposé (de beaux fruits et non *des beaux fruits) qui veut que cette règle ne s'applique pas lorsque l'adjectif constitue avec le nom un mot composé (J'ai acheté des petits fours). 
Par ailleurs, on remarque que selon la règle précédemment mentionnée, il devrait en être de même avec le déterminant partitif: "Il vend $d u$ pain" devrait donner "Il vend *de bon pain". Or, le français actuel respecte de moins en moins cette indication et préfère utiliser le pluriel: "Il vend de bons pains" ou encore conserver la forme pleine de l'article partitif "Il vend du bon pain" si bien que cette règle tend à disparaître au pluriel. Ce passage "des" à "de" s'expliquerait par la fonction d'actualisateur que prend l'adjectif antéposé et rend superflu un second marquage de l'article.

Tous les adjectifs antéposés sont en général concernés par cette règle: "de tels agissements; d'autres personnes; J'en apprends de belles et de bien bonnes sur ton compte etc.". La nature du déterminant semble être en cause. En effet, seul le pluriel semble poser des problèmes: "un tel agissement; une autre personne; J'en apprends une belle / une bien bonne sur ton compte" ne semble pas poser de difficultés.

On remarque également que c'est surtout le pluriel du quantificateur "un" qui est concerné et non pas la forme du pluriel de l'article contracté suivi de la préposition "de": "elle cueille les belles fleurs de son jardin; elle s'intéresse aux belles fleurs de son jardin (à + les)" ou encore "elle se soucie des belles fleurs de son jardin (de + les)". Aussi, le pluriel flou et imprécis de "un" et "des" doit être, devant un élément de détermination (autre, certain, tel...), ou encore peut être, devant un adjectif autre, remplacé par la forme "de".

Par ailleurs, on remarquera la différence entre les phrases suivantes: "Je voudrais un stylo" et "J'ai besoin $d$ 'un stylo" qui donnent au pluriel "Je voudrais des stylos" et "J' ai besoin de stylos" et non "J'ai besoin *de des stylos". Il ne peut donc y avoir deux morphèmes "de" à la suite, l'un de l'autre, en français si bien que "de + des $=\mathrm{de}$ ". On distinguera également le déterminant "de" dans la phrase "Je voudrais de très belles fleurs" et la préposition "de" dans l'exemple "J'ai besoin de très belles fleurs". On constate l'effacement de l'article et la réduction du déterminant à sa partie invariable "de". Cela donne sous forme de schéma:

a)

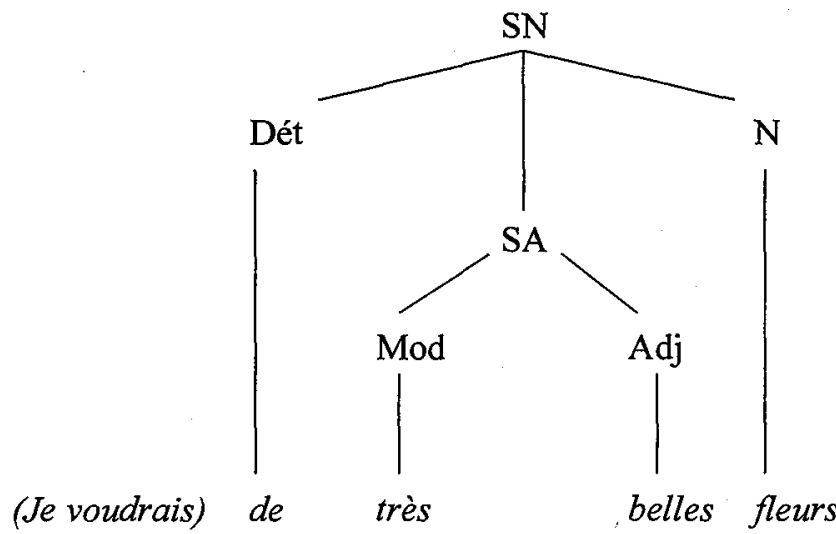




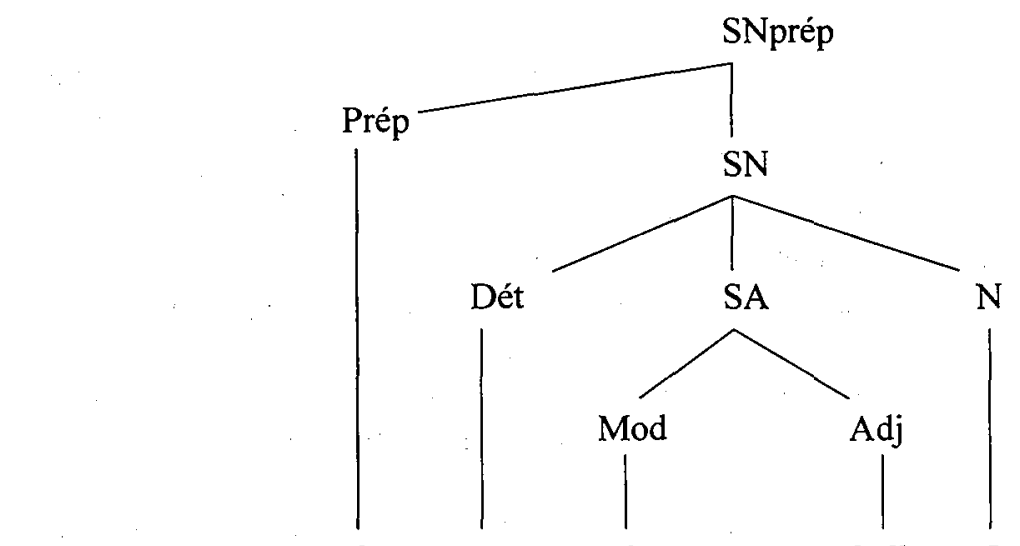

(J'aurais besoin) de très belles fleurs

$(\mathrm{SV}=$ syntagme verbal; $\mathrm{V}=$ verbe $; \mathrm{SN}=$ syntagme nominal; $\mathrm{N}=$ nom; Dét $=$ déterminant; prép $=$ préposition; $\mathrm{Adj}=$ adjectif; $\mathrm{SA}=$ syntagme adjectival; $\mathrm{Mod}=\bmod$ ificateur; $0=$ article zéro).

Aussi, si nous reprenons l'opposition vue précédemment entre le support et l'apport, on peut penser que l'adjectif antéposé au nom a la valeur du support et l'adjectif postposé prend une valeur d'apport mais cette remarque ne s'applique peut-être pas à tous les adjectifs et autres éléments déterminants. L'adjectif antéposé rappelle l'expression toute faite, connue et admise (les vastes pièces du château) tandis que l'adjectif postposé (les pièces vastes du château) caractérise, décrit ou identifie le nom. Le premier donne le thème tandis que le second apporte une information. Le premier exemple (les vastes pièces du château) rappelle une des caractéristiques de toutes les pièces du château alors que le second exemple (les pièces vastes du château) désigne un référent précis et distingue les pièces qui sont vastes de celles qui ne le sont pas. On peut donc penser que l'antéposition de l'adjectif est la marque d'un message non assumé par le locuteur, mais comprise comme allant de soi ou secondaire, tandis que la postposition de l'adjectif signale que le message de l'adjectif est assumé par le locuteur qui le considère comme un fait nouveau et premier. On peut en conclure que, devant une forme adjectivale, le pluriel flou et imprécis "des" devient la composante invariable "de" si cette forme adjectivale est un élément de détermination (autre, certain, quelque...) et, de manière optionnelle, dans les autres cas.

D'une manière générale, l'opposition "de/des" se manifeste lorsqu'un syntagme nominal est régi par le préposition "de". Elle se réalise en particulier dans les phrases suivantes: (a) "la construction de routes/la construction des routes" c'est-à-dire dans les syntagmes formés par la construction "substantif + de + substantif". Dans cet exemple, "de" s'oppose à "des" essentiellement après des substantifs à valeur collective et après des déverbaux. Le syntagme nominal représente la transformation de la 
phrase passive où le substantif sujet se présente soit avec "des" (des routes sont construites) soit avec "les" (les routes sont construites). On distingue également (b) "il parle de livres/il parle des livres", après les verbes qui se construisent avec "de". Dans ce cas, on peut considérer que "de" indique une quantité indéterminée qui s'oppose à "des" évoquant la totalité ou constituant un ensemble. Le dernier exemple (c), "il est aimé $d$ 'enfants du pays (sous-entendu "par des")/il est aimé des enfants du pays" (sous-entendu "par les"), se présente avec des compléments d'agent introduits avec "de" de sorte que l'opposition "de/des" correspond à l'opposition "par des/par les". Ces phrases sont elles-mêmes la transformation des phrases actives: "des enfants du pays l'aiment" et "les enfants du pays l'aiment". On en conclut que l'article "des" apparaît tantôt en l'absence de préposition (exemple a) tantôt après une préposition autre que "de" (exemples b et c). On assiste ainsi à effacement de l'article "des" après la préposition puisque "de + les $=$ des" et "de + des $=\mathrm{de}$ ".

\section{Conclusion}

En conclusion, il s'agit de distinguer les deux notions d'article et de quantificateur au sein de la détermination nominale. On peut noter qu'une des propriétés des quantificateurs est qu'ils possèdent un fonctionnement pluriel dans le sens où ils peuvent déterminer ou représenter le syntagme nominal. Il est clair que le constituant article ne renvoie pas uniquement à l'article indéfini. On pourrait étendre leur champ d'étude notamment aux déterminants démonstratifs ou possessifs. Quant au constituant quantificateur, il s'agit d'une forme complexe du système de la détermination qui peut également s'étendre à l'étude d'autres quantificateurs voire celle des quotificateurs (tout, chaque etc.) ou encore aux cas d'absence de détermination. Une formalisation plus précise du système de la détermination reste cependant nécessaire. Il nous reste à espérer que dans l'avenir une analyse contrastive, présentant les formes de détermination française et slovène, apparaisse afin de faciliter la tâche aux apprenants slovènes, désireux de comprendre le système français de la détermination. 


\section{Bibiographie}

BonNard, Code du Français courant, Paris, Magnard, 1997

DuBoIS J., Grammaire structurale du français: nom et pronom, Paris, Larousse, 1965

DUBoIs J. et Dubois-Charlier F., Eléments de linguistique française: syntaxe, Paris, Larousse, 1970

GRÉVISSE M., Le bon usage, Paris, Duculot, 1993

Langue française $\mathbf{n}^{\circ} 57$, "Grammaire et référence", Paris, Larousse, 1983

KLEIBER G., Article défini, théorie de la localisation et présupposition existentielle in Langue française, 57, 1983,

pp. 87-105

KLEIBER G., Sur l'anaphore associative: article défini et adjectif démonstratif in Rivista di linguistica, 1990 a, 2; 1, pp. 155-174

KLEIBER G., Article défini, unicité et pertinence in Revue romane, 1992 b, 27, 1, pp. 261-89

PnNCHON J., Morphosyntaxe du français, Paris, Hachette, 1986

PoPIN J., Précis de grammaire fonctionnelle du français, Paris, Nathan Université, 1993

RIegel M., Pellat J.-C., Rioul R., Grammaire méthodique du français, Paris, PUF, 1994

Ricardou J, Les Lieux-dits, Paris, Union générale d'éditions, 1972.

TOGEBY K., Grammaire française, Volume I, Copenhague, Berg, Merad, Spang-Hanssen, 1982

WAGNER R-L et PICHON J., Grammaire du Français Classique et Moderne, Paris, Hachette, 1991

WILMET M., La détermination nominale, Paris, PUF, 1986

WINTHER A., Description syntaxique du français, Rouen, PUR, 1996

\section{Povzetek}

\section{SAMOSTALNIŠKA DOLOČNOST: ČLEN IN KOLIČINSKI DOLOČEVALNIK}

Pričujoči članek poskuša pregledati določene pojavne oblike samostalniških besednih zvez, še posebej samostalniško določnost. Poudarek je dan na nekatere primere, ki lahko delno odgovorijo zahtevam slovenskega učenca, kajti določnost se v slovenščini izraža tudi s pomočjo sklanjatev in ne $\mathrm{z}$ uporabo člena. Zato bomo izpostavili predvsem nasprotje med členom in količinskim določevalnikom. 